\title{
Pushing the Rock up the Hill: Teaching Imaging Technology in the Classroom
}

\author{
R.B. Simmons* \\ *Department of Biology, Georgia State University, Atlanta, GA 30303
}

Teaching imaging technology in a classroom rather than a laboratory setting presents a few interesting challenges. Without having the opportunity for hands-on instruction one must find ways to bring the experience to the students. Modern technology allows the use of electronic media, which in many ways, brings the laboratory experience into the classroom. An understanding of how we came to our current place is important and I try to introduce historical information when possible. A number of concepts must be addressed to be sure that all of the students have the base line knowledge required to move from basic to more complex technologies. One immediate challenge is that many students do not have a fundamental understanding of the basic physics of light and optics. A 'memorize and forget' approach to past learning becomes apparent when dealing with senior year students who have taken the prerequisite courses in physics and chemistry but still have little to no memory of the properties of light and electrons. Hopefully by the end of the first segment of the course every student understands these concepts and we can build upon that base.

My course is broken up into distinct units, each building upon the previous so that as the semester progresses each student will hopefully be able to take the knowledge from the previous unit and apply it to the next. I begin unit one with a brief history of microscopy and photography followed by lectures on the nature of light, including the concepts of wavelength, color, energy, the photoelectric effect and different types of light sources. Next comes basic optics beginning with the nature of glass and how different types of glass affect optical properties, followed by a discussion of lenses and optical image formation. This first unit ends with a survey of image capture devices from photographic film to electronic recording media and digital management of images.

Unit two covers light microscopy and begins with the anatomy of the light microscope and illumination systems. Kohler illumination is followed by Phase Contrast and Differential Interference Contrast methods. Fluorescence technology is next and leads to wide field fluorescence microscopy applications followed by confocal and deconvolution microscopes.

Unit three covers electron microscopy and begins with systems common to both types of microscope. Vacuum systems followed by electron guns and electron optics then branch off first into transmission electron microscopy, then into scanning electron microscopy. Beam/specimen interactions lead to discussion of application of the multiple generated signals to a range of imaging and data collection modes.

Unit four looks at using concepts from the previous three sections to build even more refined imaging technologies such as the atomic force microscope and a range of medical imaging technologies.

Assessment for this course consists of written examinations at the end of the first three units and a short applied problem question assigned over the Thanksgiving break. The final examination is cumulative. 